\title{
Un caso de útero doble con embarazo doble
}

\author{
Por Jaime Caneino Bernal (:)
}

Este trabajo formó la parte principal de mi tesis de grado y hasta ahora se hace su publicación por motivos imprevistos.

Hechas las consideraciones de embriología inherentes a estos sasos de anomalías congénitas presentamos a continuación la Historia Clínica correspondiente.

Nombre: N. N.

Procedencia: Anapoima (Cundinamarca).

Natural de La Mesa (Cundinamarca)

Edad: treinta y tres años.

Estado civil: casada.

Antecidentes familiares. - Padres muertos. Cuatro hermanos: tres hombres y una mujer. La hermana ha tenido dos hijos; los embarazos y los partos en ella han sido normales. La salud de todos ellos buena.

Antecedentes personales generales. - Sin importancia.

Antecedentes ginecológicos y obstétricos.-Menarquia: quince años. Ciclo $30 \times 3$, regular, sin dolor y sin trastornos generales de importancia. Tuvo un embarazo sin complicaciones de ningún orden; parto normal atendido por comadrona. El producto de ese parto fue una niña que goza actualmente de buena salud.

Vida marital. - Tiene cuatro años de casada. Relaciones sexuales siempre normales; no hay dispareunia. Después del primer parto reaparece la menstruación a los 10 meses y el ciclo es nuevamente $30 \times 3$.

Historia actual.-La paciente ingresa al Hospital de Girarc.ot, Servicio de Maternidad, el 25 de septiembre de 1952, a las

(*) Cirujano del Hospital de San Rafael. Girardot. 
2. a. m. Es remitida por el Puesto de Salud de Anapoima con nota en la cual se advierte que ha sido controlada en el octavo mes del embarazo y tratada satisfactoriamente para pre-eclampsia leve y que el diagnóstico es: "Embarazo gemelar con retención de segundo feto por inercia secundaria".

La enferma relata que comenzó el trabajo del parto el 22 de septiembre en la noche y que fue atendida por una enfermera para un parto que culminó con la expulsión de un feto, que pesado en este hospital alcanzó 2.000 gramos y una talla de 46 centímetros, y que el alumbramiento fue normal.

A su ingreso al hospital, y practicado el examen clínico, se obtuvieron los siguientes datos: estado general: regular. Aparato cardiovascular y respiratorio: normales. El examen del abdomen nos revela lo siguiente: a la palpación en un abdomen globuloso se identifica una masa que parece corresponder a un útero em barazado y que tiene además por su lado derecho otra masa, ésta más pequeña, situada en la parte baja y a la derecha del hipogastrio. Medida la altura de la masa mayor es de 30 centímetros. En el curso del examen se aprecian movimientos fetales y se diferencian dos polos que corresponden a la porción cefálica de un feto que está colocado sobre el estrecho superior de la pelvis y el otro a la porción caudal del mismo feto que está colocado hacia el fondo uterino. A la auscultación se aprecian claramente pulsaciones cardíacas fetales con una frecuencia de 120 por minuto. En un lugar alto y hacia la izquierda de la masa se oye otra vulsación con una frecuencia que corresponde al pulso de la paciente y que permite identificar un soplo placentario.

Tacto vaginal: cuello uterino de consistencia blanda relativamente corto, con dos orificios, uno hacia la izquierda de tipo puntiforme, no deicente como corresponde a una matriz nulípa$\mathrm{ra}$; hacia la derecha el otro orificio que se toca tiene forma de hendidura ligeramente oblicua hacia atrás y a la derecha, y deja pasar un dedo hasta su orificio interno. Los fondos de saco están ocupados por una masa blanda que corresponde por una parte al útero en gestación todavía y por otra a la masa de menor tamaño, como el de una naranja, que ya se habia tocado por la parpación a la derecha y en un punto bajo. Aplicado el espéculo se ve que los orificios apreciados al tacto corresponden a dos cuellos uterinos perfectamente identificables, que están intimamente adosados y que solo los hace apreciar como individualizados un surco de poca profundidad, que está presente tanto en su parte 
anterior como en la posterior de la línea media de toda la masa que en principio se tocó como cuello único. Después de este examen se concluyó que podría tratarse de un caso de útero doble en el que ya se había hecho un parto correspondiente al embarazo de la matriz derecha y que a esa matriz en retracción correspondía la pequeña masa situada en la parte baja y a la derecha del hipogastrio, al paso que en la matriz izquierda continuaba el embarazo sin signos de trabajo, como que la paciente no manifestaba en ese momento dolores de ningún tipo y el cuello correspondiente a esa matriz estaba completamente cerrado y sin apariencia de borramiento.

Exámenes de laboratorio: N. P. N.: 39 miligramos por 100 centímetros. Orina: color amarillo, ligeramente turbio, reacción alcalina, albúmina, glucosa, pus y sangre no hay. Sedimento: células epiteliales ++ . Serología negativa.

Se procede después a hacer un examen radiológico para tratar de obtener una imagen de la presunta cavidad uterina derecha ya desocupada que contraste con la presencia del feto en la cavidad uterina izquierda. Debido a la reciente fecha del parto se encuentra contraindicado el uso de productos yodados para hacer la histerografía y por este motivo recurrí a la siguiente técnica para obtener la imagen deseada sin que el medio de contraste usado pusiera en contacto directo con el endometrio que en este tiempo se podia considerar como una herida abierta y correr en esta forma el grave riesgo de las embolías. Técnica: fijé una pequeña bolsa de caucho hecha de un preservativo, a la punta de una sonda de Nelaton. Introduje luego la sonda hasta la cavidad uterina e insuflé la bolsa con 15 centímetros cúbicos de bario; con la sonda en su posición se tomó la placa, radiológica (número 1) y como se observará dio la imagen de una cavidad uterina derecha, pequeña, correspondiente a una matriz retraída y del lado izquierdo la imagen clara de un feto en presentación cetálica. Se podría hacer una crítica a la técnica descrita por cuanto la bolsa usada podría habernos dado la falsa imagen de una cavidad perfectamente independiente de la que contiene el feto presente. Sin embargo, como se verá más adelante, se comprobó la existencia de las dos cavidades.

La conducta seguida a partir de este punto fue la de esperar a que se produjera el segundo parto espontáneamente y para esto se atendió a las siguientes razones: 1ạ El parto efectuado el 24 de septiembre podía considerarse como prematuro, ya que el 
aspecto general de la niña nacida era como de un prematuro por su peso: 2.000 gramos, su talla: 46 centímetros y su estado general. 2a En el momento la enferma no presentaba signo ninguno de trabajo de parto, y $3^{a}$ No existía sufrimiento fetal, ni alguna otra causa de parte materna ni fetal que exigiera el apresuramiento de dicho parto. En esta forma transcurrió el tiempo hasta el 30 de octubre de 1952, o sean 36 días, lapso durante el cual se le suministraron a la paciente vitaminas y hematógenos en dosis adecuada para mejorar su estado general. El anotado 30 de octubre a las 9 de la noche entra la paciente en franco trabajo para el segundo parto. A las dos de la mañana del 31 de octubre se practica un tacto que nos da los siguientes datos: orificio del cuello de la matriz derecha es aún deicente. El orificio del cuello de la matriz izquierda es regular y tiene una dilatación de $\mathbf{5}$ centímetros. La presentación es una cefálica O. I.I. A.; la bolsa de las aguas está intacta. Bajo vigilancia continua sigue la evolución del pacto normalmente y a las 2 y 55 minutos del 31 de octubre nace espontáneamente un feto de las siguientes características: sexo: masculino. Peso: 2.800 gramos. Talla: 49 centímetros. Diez minutos después se hace el alumbramiento. Placenta: 450 gramos. Cordón: 86 centímetros, inserción central.

De esta fecha en adelante la paciente entra en un puerperio sin complicaciones de ninguna clase. La involución uterina se hace correctamente y a los 14 días se procede a practicar nuevo examen radiológico para identificar las dos cavidades uterinas. Para esta oportunidad, y con las debidas precauciones, se usa la técnica corriente de la histerografía; el medio de contraste usado fue el Iodatol, del cual se inyectaron por medio de sondas de Nelaton 10 c. c. en cada una de las cavidades y se tomaron las placas que nos dan la imagen de los dos úteros perfectamente incividualizados. En esta oportunidad también se tomaron las fotografías, en las cuales se observa la posición de los dos cuellos con sus respectivos orificios. 


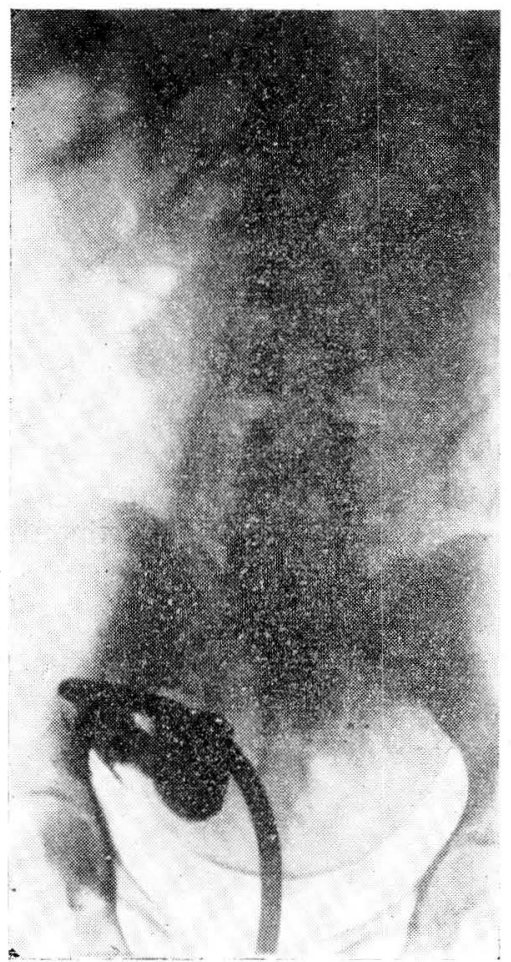

Cavidad derecha desccupada y feto en la matriz izquierda.

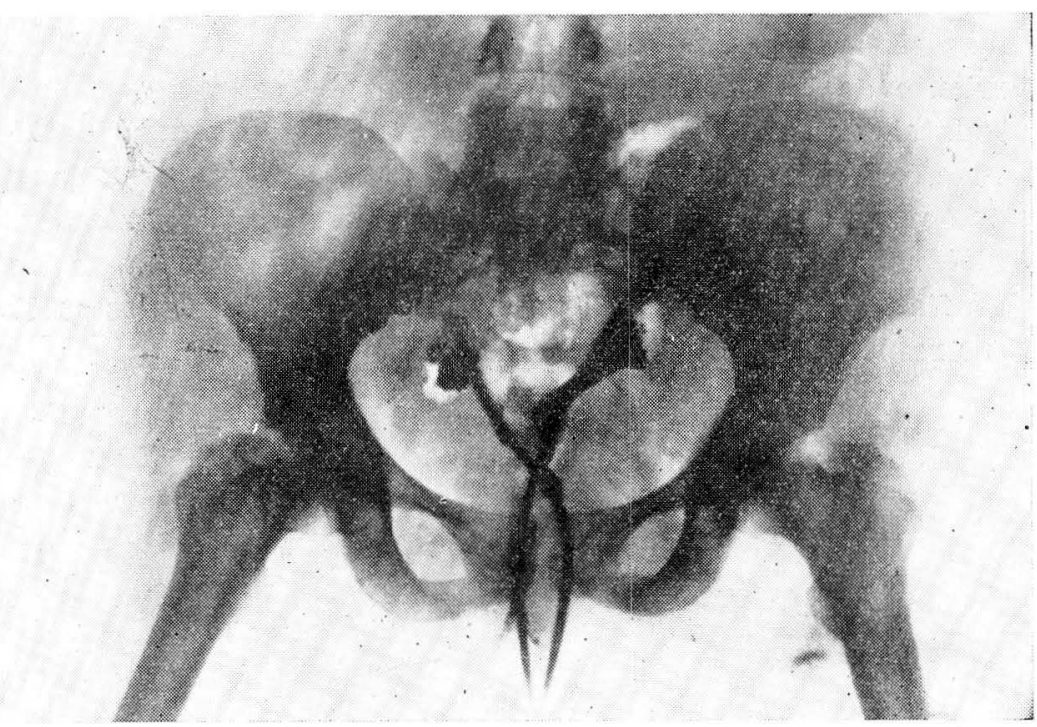

Las dos cavidades uterinas desocupadas. 

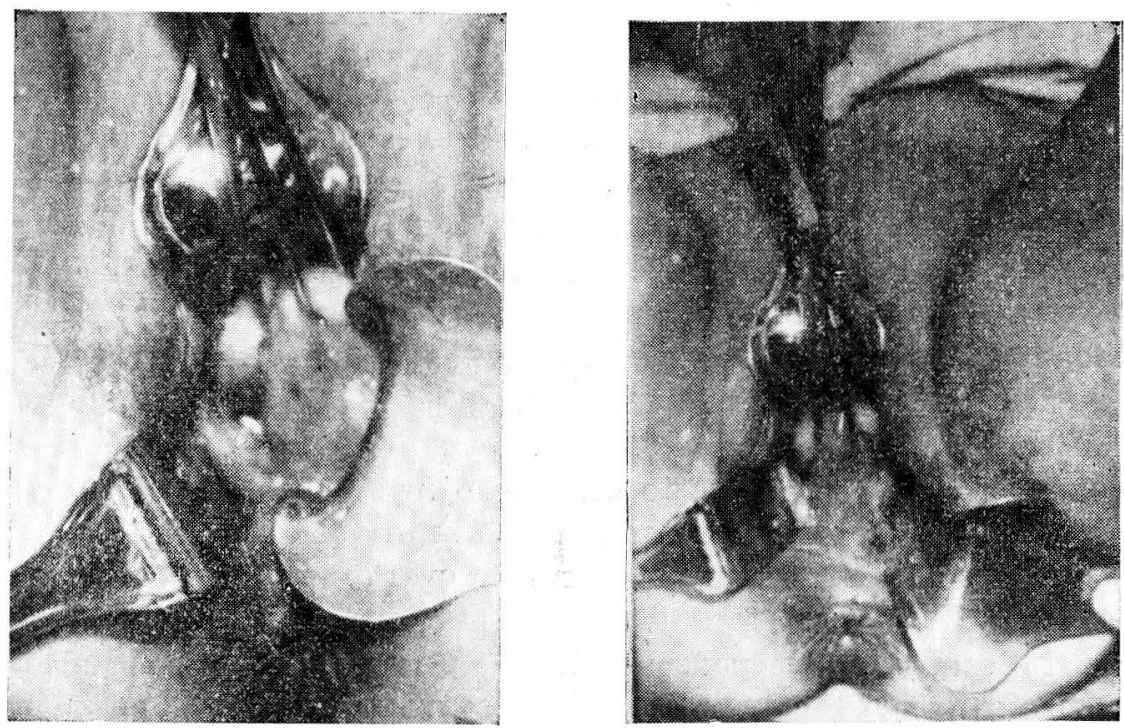

Los cueilos es án tomados cade uno con Los cuellos claramente individualizados. una pinza.

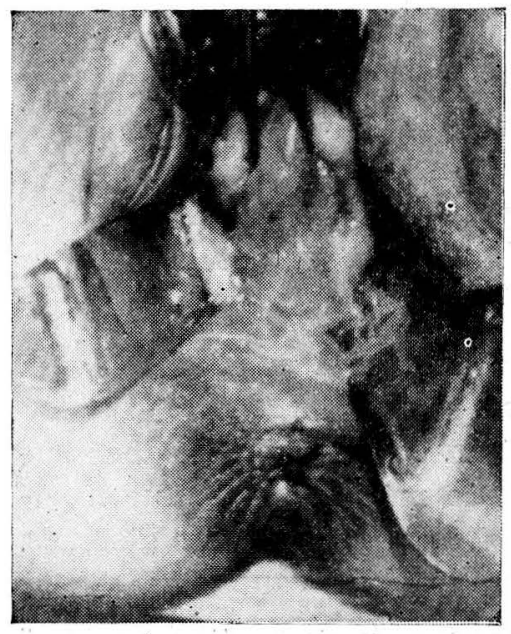

Otra vista de los cuellos.

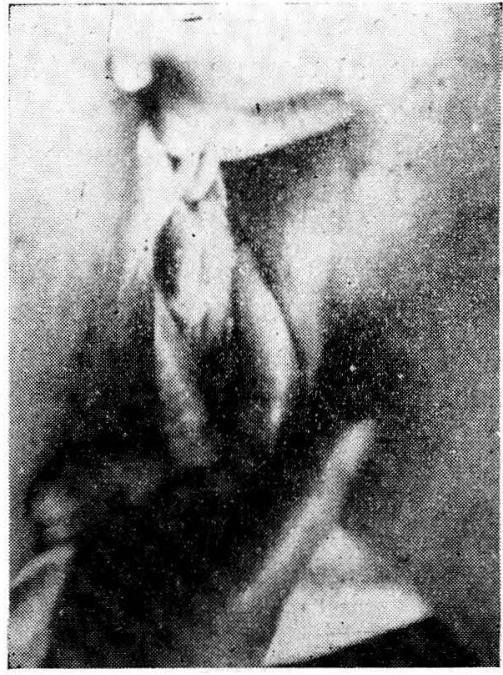

Restos de un tabique vaginal. 


\section{COMENTARIOS}

19 Constderaciones endocrinológicas.--iTiene variación la endoctrinología o se alteran las menstruaciones en estos casos de deformidad congénita? No hay razón para pensar que la armonia endocrina esté trastornada en este tipo de anomalía por cuanto a ella se refiere a una deficiencia en la unión de los conductos de Müller. Hemos de considerar de acuerdo con la frecuencia anotada por los autores, que generalmente en estos casos está repectada la integridad de la gonada.

La evolución tiene, pues, que hacerse en forma semejante a cualquier caso normal. Esto es, que lo más corriente es que el óvulo sea único o más raramente que sean varios que maduran y estallan al mismo tiempo en un solo ovario, o en los ovarios a la vez.

De este principio podemos deducir que los cambios del ciclo mensutral se efectúan bajo la influencia de un cuerpo amarillo único funcionalmente. Si el ciclo menstrual depende exclusivamente de las variaciones endocrinas sufridas por el ovario, tenemos que concluir que este ciclo se cumple en el endometrio de las dos matrices simultáneamente, o sea que la menstruación será única y con las características corrientes, solo que se cumple en una mayor cantidad de tejido endometrial. Podría compararse este caso al de las endometriosis, en donde inclusive en el tejido endometrial situado en regiones apartadas del útero se cumple, al tiempo que en éste el cambio correspondiente al ciclo ovárico.

En la paciente se cumplía el ciclo en esta forma.

2 ¿ ¿Cómo se hizo este embaraza? - Hay que recalcar aquí el antecedente obstétrico de la paciente. Tuvo ya un embarazo sin complicaciones, con parto normal. Se puede pensar que en esa primera gestación existió un óvulo único que procedente de un ovario descendió por la trompa derecha, fue fecundado en ella y se desarrolló en la matriz derecha.

En el caso de esta preñez múltiple, lo que puede haber sucedido y que debe considerarse como lo más probable, fue lo siguiente: siendo las dos cavidades uterinas perfectamente aisladas, como está comprobado, es de creer que por cada una de las trompas descendió un óvulo procedente de cada uno de los ovarios o de uno solo con migración interna de uno de los óvulos. De 
la misma manera ascendieron espermatozoides hasta cada una de las trompas y se logró la fecundación de un óvulo en cada una de ellas. Una vez obtenida esta fecundación, y al migrar los óvulos fecundados hacia la cavidad uterina se encontraron con que cada uno quedó en un útero distinto.

¿Se fecundaron los dos óvulos al mismo tiempo o en distintos ciclos, constituyendo un tipo de superfetación? Hay razones, las siguientes, que hacen pensar que la fecundación fue simultánea:

a) La superfetación, según Hartman, es extraordinariamente rara y su diagnóstico ha sido quizá confundido con embarazos generales en los que un feto marchito se ha desarrollado muy poco, al paso que el otro llega a término. La superfetación se ha visto solo experimentalmente en la coneja.

b) La ovulación es, por regla, inhibida durante la preñez en los primeros meses por el cuerpo amarillo y en los demás por las hormonas placentarias. Su inhibición es, pues, hormonal.

c) Que es sugestivo el hecho de que los partos se hubieran sucedido con un mes y ocho días de diferencia, es cierto, pero este fenómeno se explica así: el primer feto nació pequeño, de aspecto general para considerarlo prematuro, de 46 centímetros de talla y 2.000 gramos de peso. El otro fue un feto más, de acuerdo con las características de un parto a término de 2.800 gramos de peso, 49 centímetros de talla y estado general mejor. Entonces lo que sucedió es que por razones no bien determinadas se desencadenó el parto prematuro en la matriz, que ya había tenido un embarazo, al paso que en la matriz nulípara el parto fue a término. Esto nos explica la diferencia de tiempo en los partos y nos corrobora la tesis de la fecundación simultánea.

Tenemos aquí también que recordar que los fetos resultaron de sexo diferente. Esto no necesita explicación, ya que todo el proceso de este embarazo puede considerarse como un embarazo gemelar bivitelino, donde los dos fetos perfectamente pueden llenar las condiciones de este caso.

30 Complicaciones posibles en un embaraza de este tipo.-a) Las correspondientes al estado mismo de gestación. No nos interesan.

b) Las inherentes a un embarazo gemelar. No son tampoco de interés. 
c) Las que pueden presentarse por la anomalía misma: 10 El parto prematuro independiente en una matriz, tal como sucedió en nuestro caso, ya es una complicación por cuanto envuelve consecuencias para el feto. $2^{\circ}$ Posiciones anómalas, ya que la ley de Paiyot no se puede cumplir a cabalidad en estos casos, puesto que el continente no tiene exactamente la forma ovoide de su cavidad ni regularidad en su pared interna por estar en intima relación con la correspondiente pared interna de la otra matriz, que hace que las dos sean más o menos planas. 30 En el momento de parto, y en especial del segundo para nuestro caso, la matriz, que ya estaba desocupada, bien podía obrar como un tumor previo; tumor que era en ese momento de un tamaño considerable, puesto que todavía no se había hecho totalmente la involución.

40 Pronóstico para futuros embarazos.-El hecho de que en esta paciente se haya presentado un embarazo único la primera vez, y en esta ocasión uno doble sin complicaciones, nos autoriza para pensar que los próximos embarazos sean de buen pronóstico.

$5^{\circ}$ Aspecto quirúrgico.-Si se considerara que para un próximo embarazo constituyera complicación de carácter grave la presencia de dos matrices, podría procederse a la extirpación de una de ellas con la técnica corriente para histerectomía.

6 otros casos. - a) No fue posible, revisada la literatura médica nacional y mundial, encontrar casos iguales. Tan solo se citan algunos semejantes que se refieren a útero doble con embarazo simple, tópico o ectópico.

Por referencia verbal del profesor José del C. Acosta hay uno similar publicado en una revista del Hospital de San Juan de Dios, que no pudo ser consultada en ningún lugar.

El más parecido de cuyos detalles no nos pudimos enterar, es el encontrado en una referencia del Index Médicus, en donde se habla de un trabajo en el Journal Mount Sinai Hospital, 14; 807-808. Septiembre, octubre, 1954, titulado Utero doble con embarazo simultáneo, de M. E. Mintz.

\section{CONCLUSIONES}

1a Son excepcionales los casos de embarazo simultáneo en las dos cavidades de úteros dobles.

2a Entre nosotros es el segundo caso descrito, teniendo en cuenta la referencia verbal anotada o sin ella, el primero. 
3ạ En general no es fácil establecer la frecuencia de esos casos.

4ạ Con relación a la literatura mundial nuestro caso es el segundo en los últimos cinco años (Primero: Journal Mount Sinai Hospital).

\section{BIBLIOGRAFIA}

WARTON.-Ginecología.

CALAT'RONE.-Terapéutica Ginecológica.

CROSSEN y CROSSEN.-Enfermedades de la mujer.

ACOSTA HERNANDO.-Conferencia de obstetricia.

HOERNER, M. T'. WILKINSON L. H. y KAISER I. H.-Amer J. Surg. 74:100. 102 J. L. 47. Utero didelfo con gestación ectópica.

BETTINOTTI A. E.-Bol. Soc. de Obs. y Ginec. de Buenos Aires. 27: 97-103. Mayo 13. 48. Utero didelfo y embarazo.

BRODY S.-Amer J. Obst. and Ginec. 56: 555-557. Septiembre 48. Presentación transversa en útero bicorne con placenta previa.

STREAN C. J. y PORTNUFF J. C. J.-Obst. and. Ginec. Brit. Emp. 55: 41-43. Febrero 48. Complicación del trabajo en útero didelfo.

AUSENS A. W.-Nederltijảscvhr y, Gemeesk 85: 1586. Abril 12. 47. Menstruación ảurante el embarazo en útero doble.

CORTES G.-Arch. de Obst. y Ginec. 54: 258-273. Mayo-junio 49. Embarazo tubárico y útero bicorne.

SMITH W. P.-Amer. J. Surg. 74: 856-859. Diciembre 47. Cesárea siete años después de hemi-histerectomía en un útero bicorne.

MARCUR G. W. U. S.-Armed Forces N. J. 2: 1227-1229. Agosto 51. Embarazo en el lado derecho de un útero doble.. Brit. N. J. 2: 926-927. Octubre 21.52. Embarazo en un cuerno rudimentario de un útero bicorne.

HAGOOD W. J. y EASTLACK W.-Virginia M. Month 78: 255-258. Mayo 51. Embarazo y útero bicorne.

IBRUGGER A.-Zentralbl Gynak 71: 638-645. 1959. Curso del trabajo en útero seudidelfo.

LIU E. L.-Chino M. J. 67: 95-96. 1949. Caso de aborto en un cuerno rudimentario y subsecuente embarazo intrauterino en útero bicorne.

MINTZ M. E. J.-Mount Sinai Hospital. 14: 808-808. Septiembre, octubre 47. Utero doble con embarazo simultáneo.

KLEIN J.-Amer. J. Obst. and. Ginec. 63: 321-323. Febrero 47. Embarazo complicado con útero doble, cuello y vagina.

Girardot, febrero de 1959.

Doctor JAIME CANCINO BERNAL

(Cirujano Hospital San Rafael)

Carrera 12, número $18-60$ - Girardot. 